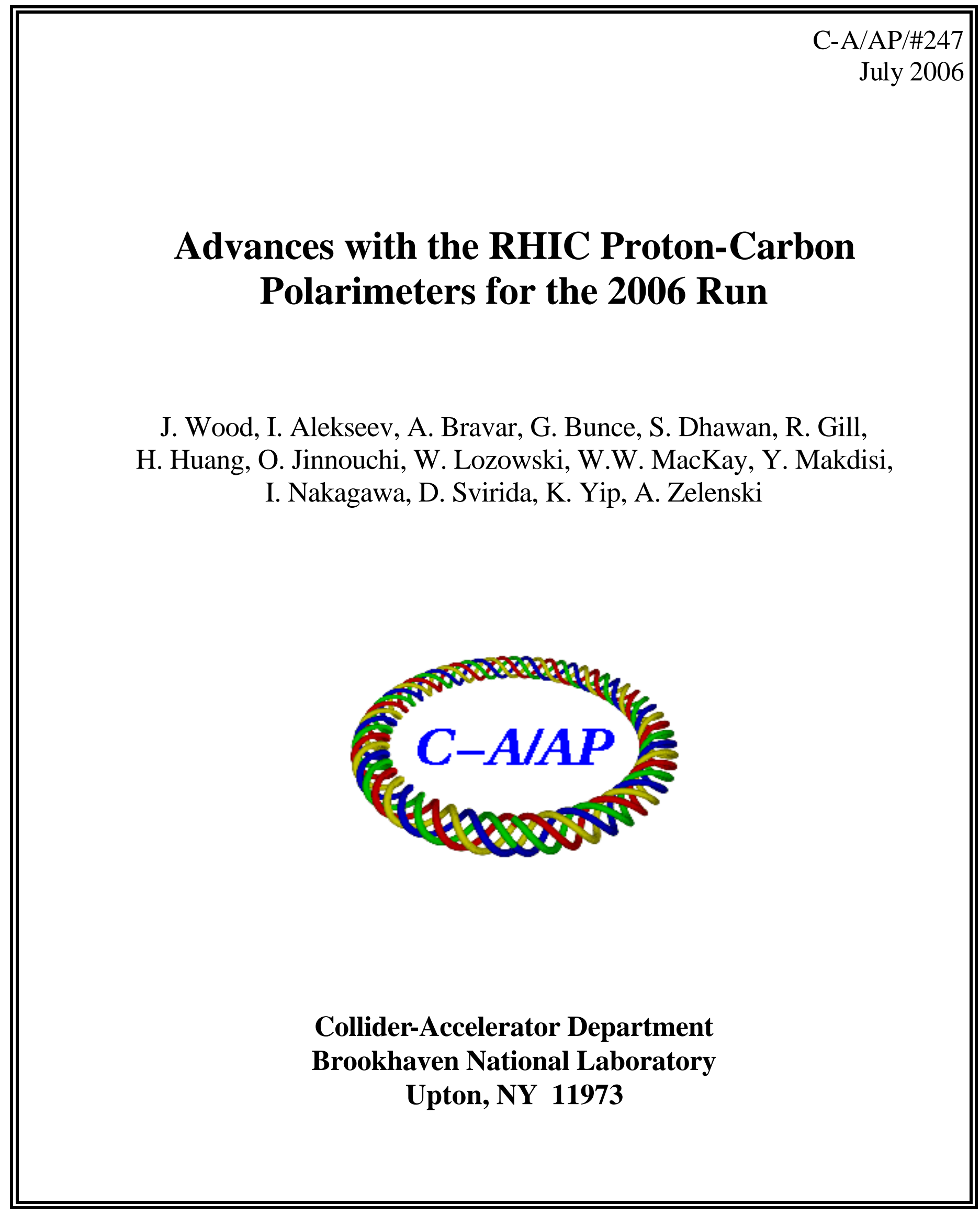




\title{
Advances with the RHIC Proton-Carbon Polarimeters for the 2006 Run
}

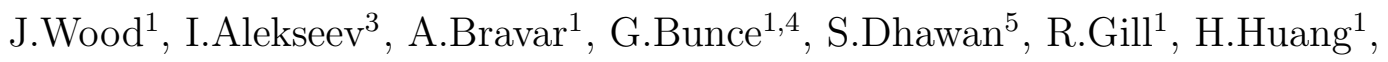 \\ O.Jinnouchi ${ }^{4}$, W.Lozowski ${ }^{2}$, W.W. MacKay ${ }^{1}$, Y.Makdisi ${ }^{1}$, I.Nakagawa ${ }^{4}$, \\ D.Svirida ${ }^{3}$, K.Yip $^{1}$, and A.Zelenski ${ }^{1}$ \\ (1) Brookhaven National Laboratory, Upton, USA \\ (2) Indiana University Cyclotron Facility, Bloomington, USA \\ (3) Institute for Theoretical and Experimental Physics, Moscow, Russia \\ (4) RIKEN BNL Research Center, Upton, USA \\ (5) Yale University, New Haven, USA
}

During the 2006 polarized proton run at RHIC, the proton-carbon polarimeters continued to serve as the primary monitors of the beam polarization. Since the installation of the polarimeters prior to the 2001-2002 run, the CNI Polarimeter Group has worked to improve the operation and functionality of these devices. For the 2006 run several new features and measurement techniques were implemented. These include improving the display of "online" polarization results, measurement of the transverse beam size, and measurement of the spin tune. This reports discusses the details of these new features for the RHIC protoncarbon polarimeters. 


\section{Online Polarization Display}

During the 2006 RHIC run, an effort was made to quickly produce and distribute a summary of each measurement from the proton-carbon polarimeters. Polarimeter measurements provide several pieces of information about the state of the beams in RHIC. Polarimeter experts can easily locate the relevant information, but prior to the 2006 run little information was accessible to polarimeter users and operators. A set of summary plots was designed to display useful results from each measurement. These so-called "online" plots are distributed to the operators using the standard network protocol at $\mathrm{RHIC}^{1}$ and are available for viewing in the RHIC Main Control Room (MCR) within seconds of a measurement. The online plots are described in further detail in the section below.

The polarimeter data acquisition (DAQ) systems are operated through a software application, called PolarControl, that is accessible to the RHIC operators in MCR. When the operators issue a command to start a measurement, the polarimeter DAQ collects data. This data is then automatically analyzed and the online plots are produced in the form of a GIF file. The online plot file is transferred to a server where it can be accessed and displayed by the PolarControl application.

For each measurement there are two pages of online plots generated. The first page contains the following plots: bunch asymmetry vs. bunch number, bunch event distribution, time of flight (tof) vs. energy of measured events, detector event distribution, and the event rate vs. measurement time. An example of the first page of online plots is shown in Figure 1.1. The second page of online plots contains: ratio of up/down events vs. detector position, measured polarization vector, and the values of several measured asymmetries. An example of the

\footnotetext{
${ }^{1}$ The Controls Group in the Collider-Accelerator Dept. at BNL make use of the CDEV protocol to communicate information from various devices at RHIC.
} 
second page of the online plots is shown in Figure 1.2. The contents of each plot are described below.

\subsection{Bunch asymmetry vs. bunch number}

An asymmetry is calculated for events from each individual bunch. The polarization bunch pattern is used to determine if each bunch is either spin up or spin down. The spin up bunches are plotted as red points. The spin down bunches are plotted as blue points. If the beam is polarized, a negative asymmetry is expected for spin up bunches (red points) and a positive asymmetry for spin down bunches (blue points). If the red and blue points in the plot do not have the expected sign, then the polarization pattern may not have been properly loaded. The polarimeter DAQ system reads the pattern from a device manager when each measurement begins. Infrequently, an incorrect pattern, which does not actually correspond with the beam in the rings, is sent to this device manager.

The asymmetry that is calculated for each bunch gives a measure of the difference in yields for the left and right sides of the polarimeter. This is referred to as an asymmetry in the $\mathrm{X}$ direction. By convention, the $\mathrm{X}-\mathrm{Y}$ plane is the plane transverse to the beam direction. The $\mathrm{X}$ axis lies along the horizontal direction, and the $\mathrm{Y}$ axis along the vertical direction. The left-right $\mathrm{X}$ asymmetry for the $i^{\text {th }}$ bunch is defined as,

$$
\varepsilon_{i}^{L R}=\frac{N_{i}^{L} \mathcal{A}-N_{i}^{R}}{N_{i}^{L} \mathcal{A}+N_{i}^{R}},
$$

where $N_{i}^{L(R)}$ is the carbon event yield for the left(right)-side detector(s) from the $i^{t h}$ bunch. $\mathcal{A}$ represents the ratio of total right events for all bunches to the total left events for all bunches.

$$
\mathcal{A}=\frac{\sum_{i=1}^{120} N_{i}^{R}}{\sum_{i=1}^{120} N_{i}^{L}} .
$$

Equation 1.1 is used to calculate two different asymmetries for each bunch, one using only the $90^{\circ}$ detectors $\left(\varepsilon_{i}^{L R 90}\right)$ and one using only the $45^{\circ}$ detectors $\left(\varepsilon_{i}^{L R 45}\right)$. For much of the 2006 run, $\varepsilon_{i}^{L R 90}$ was used in the online plots. After May 31, an average $\mathrm{X}$ asymmetry was calculated using both $\varepsilon_{i}^{L R 90}$ and $\varepsilon_{i}^{L R 45}$. Including 
the $45^{\circ}$ detectors improved the statistical uncertainty of these asymmetries. The average $\mathrm{X}$ asymmetry, $\varepsilon_{i}^{\text {ave } X}$, is given by

$$
\varepsilon_{i}^{a v e X}=\frac{N_{i}^{90} \varepsilon_{i}^{L R 90}+\frac{N_{i}^{45}}{2} \sqrt{2} \varepsilon_{i}^{L R 45}}{N_{i}^{90}+\frac{N_{i}^{45}}{2}},
$$

where $N_{i}^{90(45)}$ is the event yield for all of the $90^{\circ}\left(45^{\circ}\right)$ detectors. The statistical error of $\varepsilon_{i}^{a v e X}$ is given by

$$
\sigma_{i}^{a v e X}=\frac{1}{\sqrt{N_{i}^{90}+\frac{N_{i}^{45}}{2}}}
$$

Equations 1.3 and 1.4 are used to calculate the asymmetries and errors shown in Figure 1.1. The asymmetries are plotted versus the bunch number.

\subsection{Bunch event distribution}

This plot displays the total number of detected carbon events versus bunch number. The number of detected events are plotted on a log scale. The event yield reflects which bunch numbers are actually filled with a proton bunch.

\subsection{Time of flight vs. energy (banana plot)}

This plot shows the measured time of flight versus energy for all events detected by the polarimeter. The measured time of flight for each event must be corrected for an offset between the polarimeter timing signal and the actual time that a proton bunch interacts with the target. The measured time of flight, to $f_{\text {meas }}$, is corrected by a value, $t_{0}$, to give the corrected time of flight to $f_{c o r}$.

$$
\text { to } f_{\text {cor }}=\text { to }_{\text {meas }}-t_{0}
$$

The $t_{0}$ value is adjusted individually for each of the 72 polarimeter detector strips. The $t_{0}$ values can vary by $\sim 1$ ns from strip to strip. 
The data shown in the online plot show the measured time of flight $\left(t o f_{\text {meas }}\right)$ versus the measured kinetic energy $\left(E_{k i n}\right)$. The tof-energy correlation is clearly seen in the data. The blue line on the plot represents the expected tof-energy correlation for scattered carbon nuclei, given by,

$$
\text { tof } f_{\text {meas }}=d \sqrt{\frac{m_{C}}{2}} \frac{1}{\sqrt{E_{\text {kin }}}}+\left\langle t_{0}\right\rangle,
$$

where $d=18 \mathrm{~cm}$ is the distance from the carbon target to the detectors and $m_{C}=11.2 \mathrm{GeV} / \mathrm{c}^{2}$ is the carbon mass. $\left\langle t_{0}\right\rangle$ is the average $t_{0}$ value for the 72 detector strips. $\left(\left\langle t_{0}\right\rangle_{B L U E} \approx-11 n s,\left\langle t_{0}\right\rangle_{Y E L L O W} \approx-16 n s\right.$. $)$ The red lines on the plot represent the boundaries for selecting carbon events. The red lines follow the carbon tof-energy correlation (Equation 1.6) $\pm 15 n s$ for $400 k e V<E_{k i n}<$ $900 \mathrm{keV}$.

\subsection{Detector event distribution}

This plot displays the number of detected carbon events in each of the polarimeter's six silicon detectors. All detectors are expected to see a similar number of events. A low event count for a particular detector could indicate a problem. However, when using a horizontal target, the target frame shadows detectors 2 and 5 (the $90^{\circ}$ detectors). So, detectors 2 and 5 should see few events with a horizontal target.

\subsection{Event rate and target motion}

For each measurement, the number of detected events is determined every second. The event rate (events/sec) is plotted versus the measurement time. Also, superimposed on this plot is a histogram of the target motion. Each second of the measurement, the target position is read from the target motor. The red line shows the target position versus measurement time. In the example shown in Figure 1.1, the target is scanned through the beam, and the shape of the beam profile is reflected in the event rate histogram. The peak target position of the 
profile is found by fitting the event rate histogram with a Gaussian function. The peak position is indicated beneath the plot.

\subsection{Ratio spin up to spin down vs. detector position}

This plots shows the ratio of detected events from spin up bunches to that from spin down bunches. The ratio, $R_{i}$, which is calculated for each detector separately, is given by,

$$
R_{i}=\frac{N_{i}^{\uparrow} / N_{i}^{\downarrow}}{N_{t o t}^{\uparrow} / N_{t o t}^{\downarrow}},
$$

where $i$ is an integer from 1 to 6 , representing the six detectors. $N_{i}^{\uparrow(\downarrow)}$ is the

number of events from spin up (down) bunches in the $i^{t h}$ detector. $N_{\text {tot }}^{\uparrow(\downarrow)}$ is the number of events from spin up (down) bunches in all six detectors.

The ratio, $R_{i}$, is plotted versus the $\phi$ angle detector position in degrees. This ratio gives a measure of the analyzing power $A_{N}$, and therefore the ratio shows the sinusoidal $\phi$ dependence of $A_{N}$. The ratio is fit with the following function,

$$
f(\phi)=\text { Const }+ \text { Amp } \times \sin \left(\phi+\phi^{\prime}\right) .
$$

Const, Amp, and $\phi^{\prime}$ are all free parameters in the fit. The values of these parameters and the $\chi^{2}$ per degree of freedom are all displayed on the plot.

\subsection{Polarization vector}

The last online plot shows the measured polarization vector, which is determined from measured $\mathrm{X}$ and $\mathrm{Y}$ asymmetries. The value of the $\mathrm{X}$ asymmetries are plotted on the vertical axis and the $\mathrm{Y}$ asymmetries on the horizontal axis. The shaded blue area represents the area contained within the error of the "X fit" and "Y fit" asymmetries. The $\mathrm{X}$ fit and $\mathrm{Y}$ fit asymmetries are based on sinusoidal fit to the asymmetries from all six detectors. (Note: The X fit and Y fit are not determined from the same fit to the ratio in the previous plot.) Asymmetries using other combinations of detectors are also displayed on the plot. The errors 
from the $\mathrm{X}$ asymmetries using the $90^{\circ}$ and $45^{\circ}$ detectors are represented by the blue and red lines respectively. The green lines show the $\mathrm{Y}$ asymmetry with the $45^{\circ}$ detectors. The pink lines represent the asymmetry between detectors 1 and 4 , and the black lines show the asymmetry between detectors 3 and 6 . The values of some of these asymmetries are displayed to the right of the plot. The average $A_{N}$ value for the measurement is also shown. The average $A_{N}$ is determined by weighting previously measured $A_{N}$ by the currently measured $|t|$ distribution. The online polarization value (displayed at the top of the page) is given by the $\mathrm{X}$ fit asymmetry divided by the average $A_{N}$. 

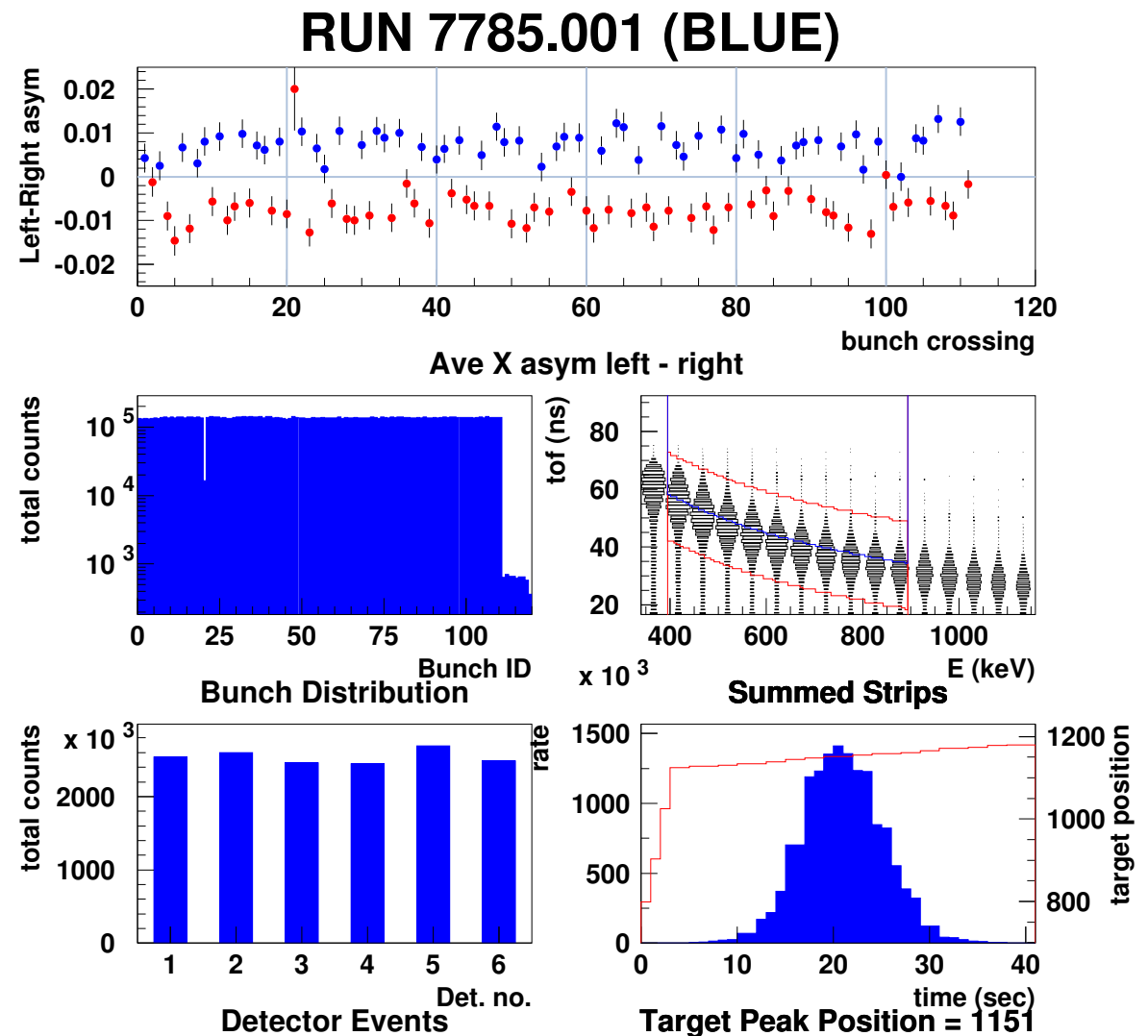

Figure 1.1: Example of the first page of the online plots display. The polarimeter run number and RHIC ring (BLUE or YELLOW) are displayed at the top of the page. The figure also contains the following plots: bunch asymmetry vs. bunch number, bunch event distribution, time of flight (tof) vs. energy of measured events, detector event distribution, and the event rate vs. measurement time. 

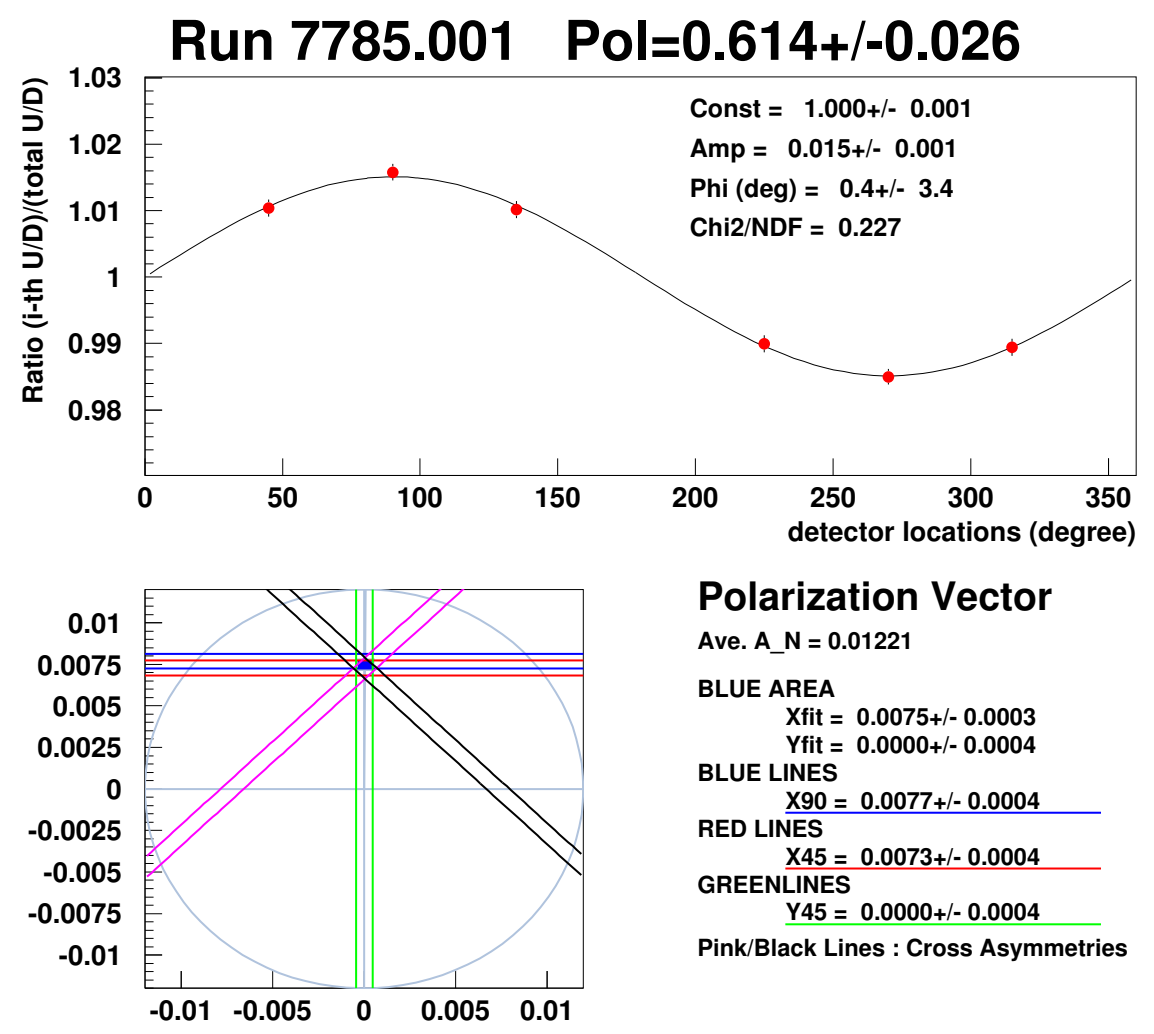

Figure 1.2: Example of the second page of the online plots display. At the top of the page, the polarimeter run number, the measured polarization, and statistical error are shown. This page shows plots of the ratio of spin up to spin down events vs. detector position and the measured polarization vector. In the lower-right corner, the average $A_{N}$ and some measured asymmetries are displayed. Polarization is given by the "X fit" asymmetry divided by the average $A_{N}$. 


\section{Beam Emittance Measurement}

A new measurement technique was developed during the 2006 run to measure the transverse beam emittance. The transverse emittance is defined as the phase space of the beam in the transverse plane. To measure the emittance, the polarimeter carbon target is passed through the beam. The scattered carbon events detected by the polarimeter are then used to make a profile of the beam shape. The width of the beam profile can be related to the beam emittance. The RHIC polarimeters use both vertical and horizontal oriented targets. For horizontal emittance measurements, a vertical target is stepped through the beam with a horizontal motion. In the same way, horizontal targets are used to measure the vertical beam emittance.

\subsection{Description of measurement technique}

In order to measure the beam emittance, the polarimeter target position must be carefully tracked as it is moved through the beam. The polarimeter targets move in discrete steps of $0.11 \mathrm{~mm}$. For each step the target motor produces a signal. These target signals are processed by the polarimeter DAQ system and written into the data stream in the same way the that scattered carbon events are. By noting when these target signals occur, they can be used to relate the carbon events to a specific target position. This is achieved by associating each detected event with a beam revolution number. The RF clock from the accelerator is used to derive the number of revolutions that the beam has made during a measurement.

There are two signals from the target motor that are used for tracking the target position. One signal is issued for each step that the target moves. A second signal is issued only when the target moves in one direction. When an 
emittance measurement is made, the target moves through the beam in one direction, then back through the beam in the opposite direction. The step signals and the direction signals can then be used to recreate the location of the target. Figures 2.1 and 2.2 show examples of accumulated step and direction signals during emittance measurements. Fig. 2.1 shows that the direction signal is detected as the target moves into the beam. For the measurement in Fig. 2.2, the target was on the opposite side of the target frame, so the direction signal is present as the target moves back to its home position.

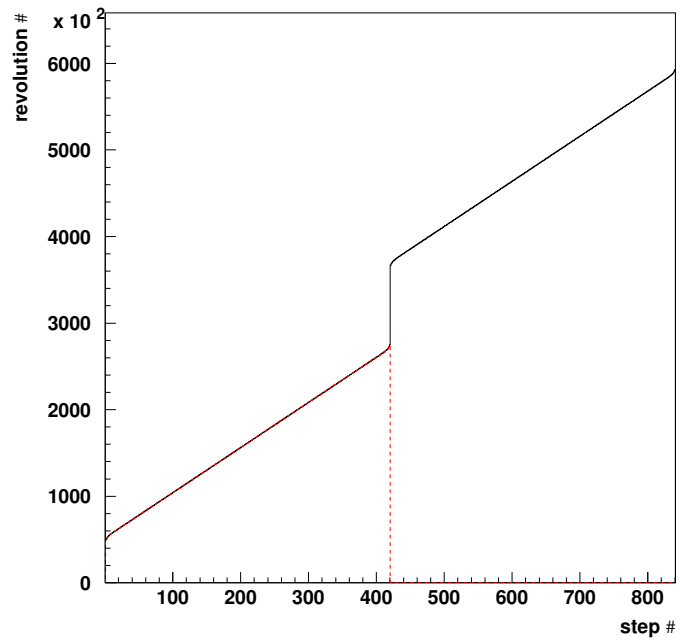

Figure 2.1: The horizontal axis shows the number of step signals accumulated by the polarimeter DAQ system. The vertical axis shows the beam revolution number when each step occurred. (The revolution period in RHIC is $\sim 12 \mu s$.) The dashed red line represents the direction signals detected by the polarimeter DAQ. At the $420^{\text {th }}$ step, the target stops briefly, changes direction, and then returns to the starting position. The direction signal is present for the first half of the target motion. This indicates that one of the odd-numbered targets was used: 1,3 , or 5 .

As the carbon target is moved through the beam, scattered carbon events are detected and stored by the polarimeter DAQ system. Each of these events is associated with a beam revolution number, and the revolution number can relate 


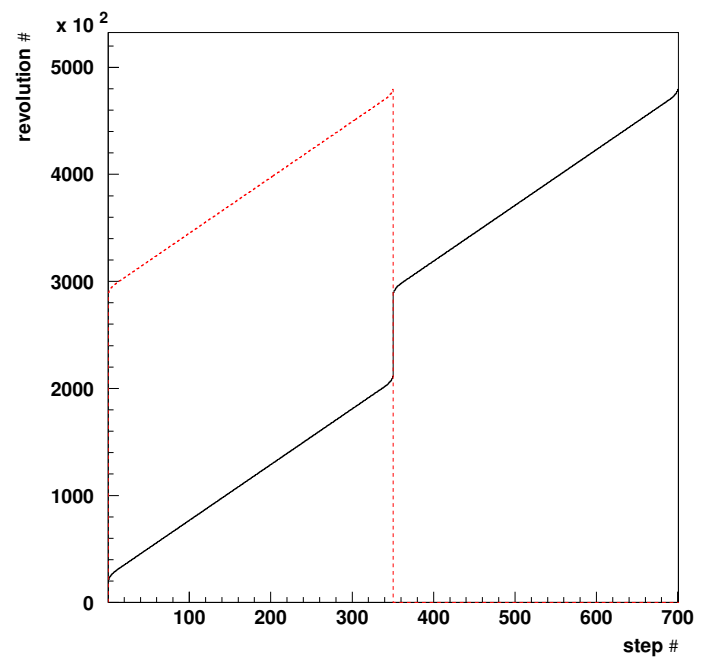

Figure 2.2: The horizontal axis shows the number of step signals accumulated by the polarimeter DAQ system. The vertical axis shows the beam revolution number when each step occurred. (The revolution period in RHIC is $\sim 12 \mu s$.) The dashed red line represents the direction signals detected by the polarimeter DAQ. At the $350^{\text {th }}$ step, the target stops briefly, changes direction, and then returns to the starting position. The direction signal is present for the second half of the target motion. This indicates that one of the even-numbered targets was used: 2,4 , or 6 . 
each event to a corresponding target position. Figure 2.3 shows the number of detected events versus target steps from a measurement using a vertical target. The target crosses the beam twice, as it moves into the beam and back out to the start position. Thus, the two resulting peaks represent two measurements of the horizontal profile of the beam.

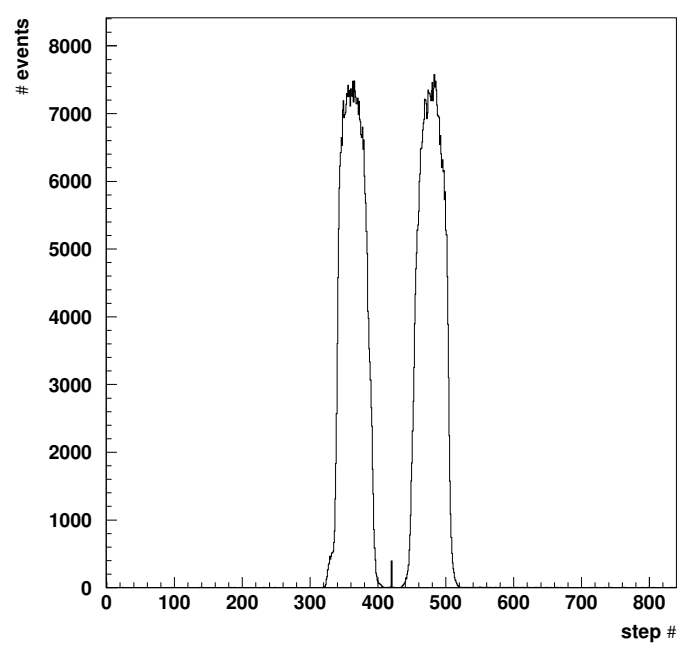

Figure 2.3: The number of detected events versus the step signals from the target. The carbon target moving into the beam and then back out to the start position results in two profile peaks. The data shown here corresponds to the target motion shown in Fig. 2.1. The target stops and reverses direction at step 420.

The analysis software for the emittance measurement is designed to select only one of the two profile peaks to determine the width of the beam. By default the second peak is used. The analysis algorithm finds the approximate center of the peak by locating the target location with the highest event rate. Then, a range of \pm 50 steps is selected for fitting with a Gaussian function. The fit results are automatically logged, so that they can later be accessed by accelerator physicists. For each measurement, there are four values that are logged from the fit: the peak center position ( $\mu$ from the Gaussian fit), the beam width $(\sigma$ from the fit $\times 0.11$ $\mathrm{mm} / \mathrm{step})$, the relative width $(\sigma / \mu)$, and the peak center position divided by the total number of events in the peak. 
The measured profile peak includes events from all of the proton bunches circulating in the ring. Under typical running conditions, there are 111 bunches stored in each of the RHIC rings. The detected events can be separated to study the profile shape of each bunch individually. The four parameters described in the previous paragraph are also calculated and logged for each bunch in the ring. In addition to logging all of these numbers, a set of summary plots is sent to a central server where it can be accessed and displayed in the MCR. The summary plots from an example measurement are shown in Figure 2.4. The summary figure includes plots of the peak position versus bunch number and the beam width versus bunch number. The y-axis projections of the peak positions and beam widths are also shown. At the bottom of the page, the profile peak, including events from all bunches, is displayed. The resulting function from a Gaussian fit is superimposed on the plot, and the fit parameters are shown to the right of the plot. The time to move the target and collect the data for this type of measurement is usually less than 10 sec.

\subsection{Example of encountered problems}

The emittance measurements with the proton-carbon polarimeters have become very useful tools for monitoring the beam shape in RHIC. However, there are some problems that have been encountered using this technique. The most common problem that is observed is measuring a non-Gaussian shaped profile. There could be several reasons why the measured profile is non-Gaussian. Some possible explanations could be: background events not coming from the target, vibration or misalignment of the target, or the beam may actually be non-Gaussian shaped. Regardless of the cause, a strangely shaped profile causes problems for the analysis software because a Gaussian shape is always assumed for fitting the profile. Forcing a Gaussian fit to a non-Gaussian profile shape can misrepresent the actual measured beam width (see Fig. 2.5 for an example) or the fit may altogether fail to converge to reasonable parameter values. When a strange shaped profile is observed, some further study may be required to determine the cause. Possible 


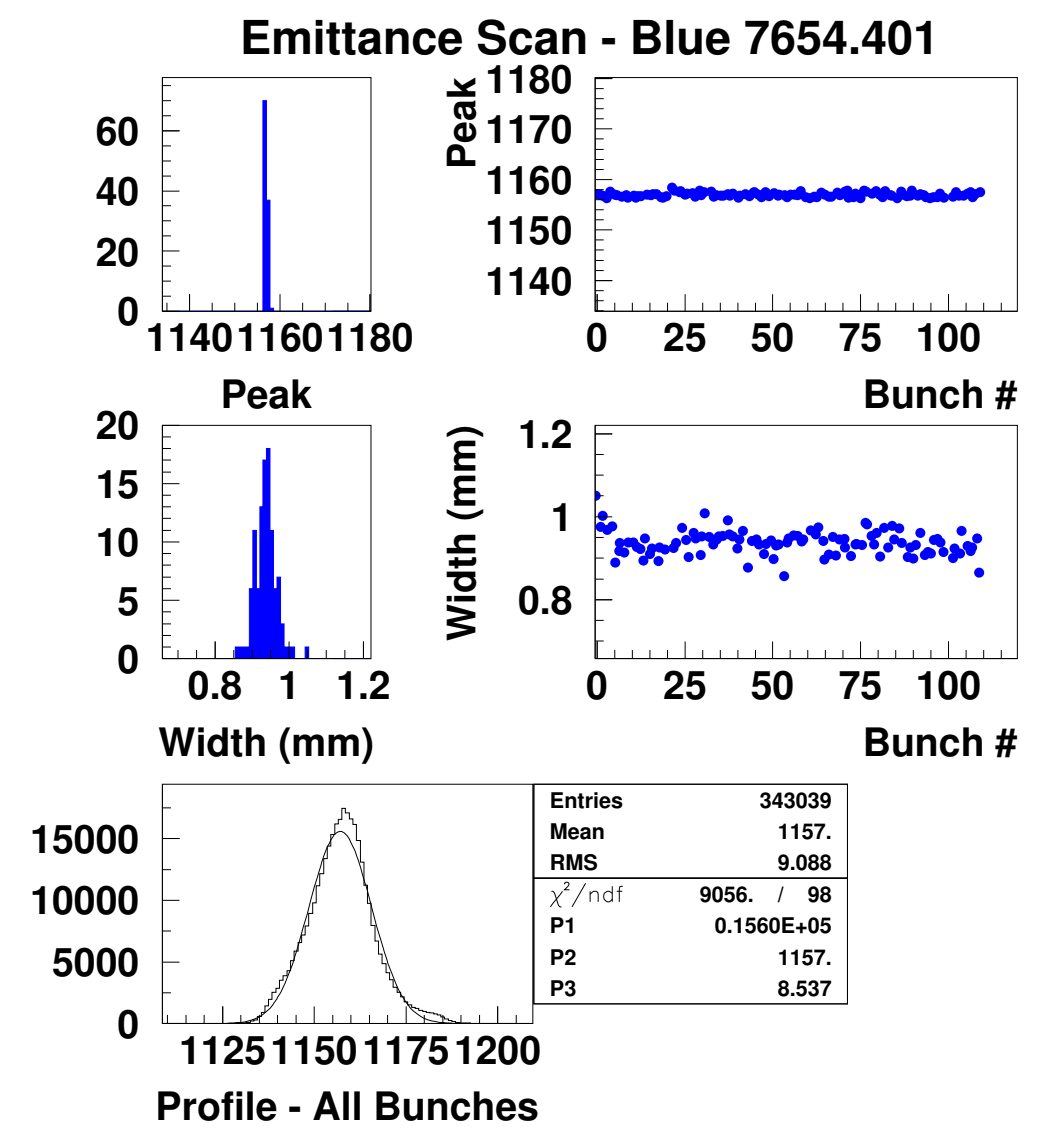

Figure 2.4: Example of the summary figure produced for each emittance measurement. At the top-right of the page is a plot of the peak position versus bunch number. To the left of that plot is the y-axis projection of the peak positions. In the middle of the page is the beam width versus bunch number and the y-axis projection of the bunch widths. The profile peak, including events from all bunches, is shown at the bottom of the page. A Gaussian fit function is superimposed on the plot. The histogram statistics and the fit parameters are displayed to the right of the profile plot. 
ways to correct this problem may include using a different polarimeter target or adjusting the beam conditions.

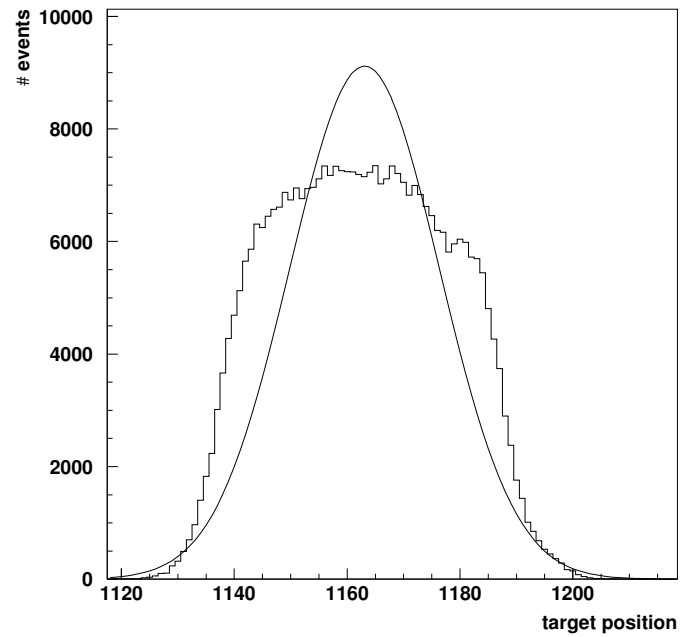

Figure 2.5: A non-Gaussian horizontal profile peak measured with a vertical target. A Gaussian function is superimposed on the plot. Flattened peaks, like the one shown here, were often observed during the 2006 run. These strange profile shapes appeared to be related to specific targets. However, the exact cause of this shape is unclear.

During the 2006 run, measurements with targets in the yellow ring were particularly problematic. Some apparent background events frequently distorted the measured profile shapes in yellow. Figure 2.6a. shows an example of such a measurement with a yellow target. Both of the measured profile peaks have a strange, non-Gaussian shape. The shape is not consistent between the two peaks. Also, background events are detected between the two peaks. These characteristics make analyzing the profile shape difficult. These problems were not always present, but they did occur more often with the yellow polarimeter than with blue.

Another complication with the emittance measurements with the yellow polarimeter is an unexplained offset in the position of the measured profile peaks. The position of the second peak does not appear at the same target position 
as the first peak. This is illustrated in Figure 2.6a. For this measurement the target moved 350 steps into the beam, then moved 350 steps back to the starting position. The event spike in Fig. 2.6a. at step 350 indicates that the target rested there briefly before changing directions. For this measurement (with an even-numbered target), the target position is given by:

$$
\begin{aligned}
& \text { targetposition }=\text { startposition }- \text { step } \quad(\text { step } \leq 350) \\
& \text { targetposition }=\text { startposition }- \text { totalsteps }+ \text { step }(\text { step }>350) \text {. }
\end{aligned}
$$

For this measurement, startposition $=1900$ and totalsteps $=700$. From the location of the peaks in Figure 2.6, the first peak suggests a target position of $\sim 1570$, while the second peak gives a position of $\sim 1605$. Other methods of determining the target position indicate that the first peak reflects the true target location and the second peak is offset by approximately 30 steps. The origin of this offset is not understood. The offset was always present for emittance measurements with all the yellow targets, both vertical and horizontal. The offset was never observed with the blue targets.

In addition to the issues described above, one other problem plagued the emittance measurements in both the blue and yellow rings. The direction signals from the target motor were often not detected by the polarimeter DAQ. If the direction signals are not received, then the analysis software cannot determine when the target was moving into the beam and when it was moving back out of the beam. Nonetheless, the analysis software can still provide a useful emittance measurement. Even if the direction signal is not present the fitting algorithm will still usually locate and determine the width of one of the measured profile peaks. However, the location of the peak target position may not be recorded properly. The reason why the direction signals are sometimes not detected is not clear. One possible explanation is that the signal timing is outside of the window where the DAQ system looks for events. The direction signals arrive at the polarimeter DAQ 15ns later than the step signals. The step signals are nearly always detected by the DAQ, but the direction is often missed. Adjusting the timing of the direction signals with delay cables is one possible solution.

Despite the problems described above, the emittance measurements with the 

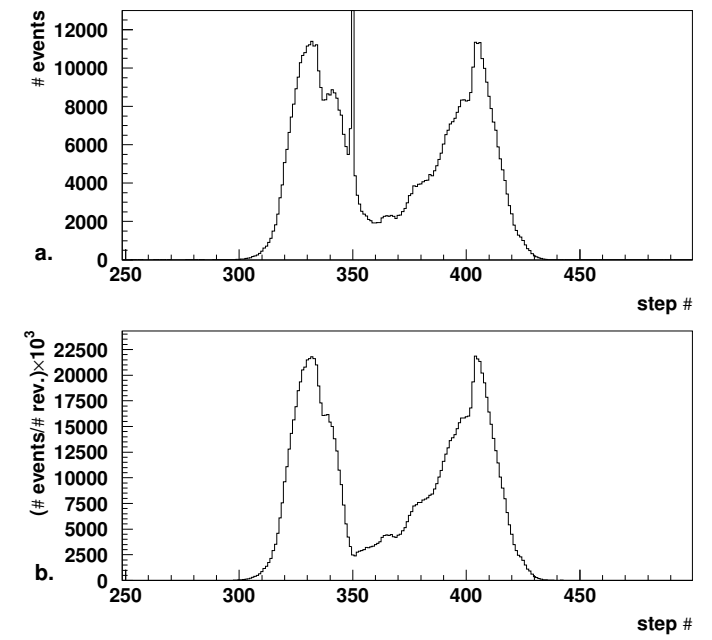

Figure 2.6: a. The number of detected events versus target step. This measurement was made with a vertical target in the yellow ring. The peak shapes exhibit a strange structure, and some background events appear in between the two peaks. There is a spike at step 350 because the target stops there before changing direction. $\mathbf{b}$. The number of detected events normalized by the number of beam revolutions at each step versus the step number. The spike at step 350 disappears when normalized. However, the strange peak shapes and background are still present after normalizing the events. 
RHIC polarimeters were successfully implemented during the 2006 run. These measure were able to quickly provide information about the size and shape of the beam profile. After demonstrating that this technique worked, the emittance measurements soon became part of the standard operation at RHIC. Emittance measurements were routinely made every 3 hours (or even more frequently) during each RHIC store. The polarimeter emittance measurement will continue to be a useful source of information for improving and monitoring the beams at RHIC. 


\section{Spin Tune Measurement}

During the 2006 run, studies were made regarding a new method for measuring the RHIC spin tune using the proton-carbon polarimeters. The spin tune is defined as the number of precessions that the polarization vector makes per revolution around the RHIC ring. A precise measurement of the spin tune could be very useful for determining the optimum settings for the RHIC Siberian snake magnets. The measurement technique, proposed by W. W. MacKay, makes use of the polarimeter to determine a spin asymmetry for each revolution, or turn, around the ring. The specific requirements for the polarimeter measurement are described in more detail below.

This proposal for determining the spin tune suggests using the polarimeter to measure a turn-by-turn spin asymmetry. The polarimeter typically sees less than one scattered carbon event for each bunch passing the carbon target. (See Figure 3.1.) So, the events from a single beam revolution do not yield a statistically significant asymmetry measurement. However, the spin tune can be determined by applying an FFT algorithm over several thousand turns to look for periodic oscillations in the measured asymmetry. Based on simulation studies, the statistics necessary to observe a definite FFT signal can be acquired by repeating this measurement with a reasonable number of bunches $(<\sim 100)$.

One of the challenges in doing this measurement is to order the events detected by the polarimeter. When a polarimeter measurement is completed, the collected events are written to a data file, but they are not written in the chronological order that they were detected. A software program was developed to loop over the events in the data file and reorder them according to the turn number that is associated with each event. As the data are reordered, the program also computes a simple asymmetry for each turn. The program sums the number of events in every turn, assigning +1 to each event scattered to the right and -1 to events 


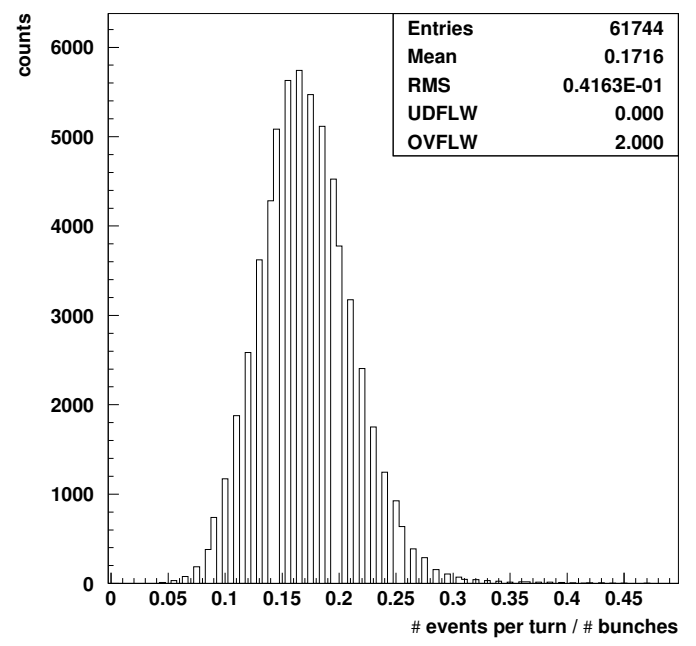

Figure 3.1: The number of detected events for each bunch passing the carbon target. The number of detected events is summed for each beam revolution, then divided by the number of bunches in the ring. This data is from a typical measurement using a vertical target with 111 bunches in the blue ring. The beam intensity was approximately $1 \times 10^{11}$ protons per bunch. The target position was fixed to give the maximum event rate throughout the measurement. The event rate can vary for different-sized targets. The targets most frequently used in the RHIC rings produce rates of $<0.4$ events per bunch passing. 
scattered to the left. Events in the $45^{\circ}$ detectors are scaled by $1 / \sqrt{2}$. Also, other detector combinations can be created to study up-down or cross asymmetries. A simple "banana" cut $( \pm 15 n s)$ is applied to the data to discard non-carbon events, but no restriction is placed on the event energy. Once an asymmetry is determined for every turn number, the values can be fed into the FFT algorithm.

An additional necessity for this measurement is to know exactly when the proton beam begins to circulate in the ring. To achieve this a signal is sent to the polarimeter DAQ whenever a proton bunch is injected into the ring. This injection signal can be obtained from the V124 module located at the 12 o'clock $\mathrm{RHIC}$ interaction region. The injection signal is routed to the polarimeter DAQ and processed in the same way as the carbon event pulses. (This very is similar to the way that the target motion signals are handled.) The injection signal is sent to a specific channel in the DAQ system. When an event is detected on this channel, a turn number is associated with the event. This turn number signifies the start of the measurement. The carbon target is moved into position prior to beam injection, so that scattered events can be accumulated immediately after the injection event.

Some dedicated time to test this measurement technique in the RHIC blue ring was allotted in the afternoon of 17 May 2006. The original plan for this measured called for injecting beam into RHIC with the polarization vector in the radial direction, but extracting this beam condition from the AGS was not possible. Instead, the beam was injected with vertical polarization, and the spin rotator magnets were tuned to cause the spin direction to oscillate between radial and vertical each turn. This gave an average polarization vector that was tilted at $45^{\circ}$. A standard polarization measurement confirmed this. A significant asymmetry was measured between detectors 1 and 4, and no asymmetry was seen between detectors 3 and 6. The measured 1-4 asymmetry corresponded to a polarization of approximately $20 \%$. Subsequent polarization measurements showed that the beam polarization decayed to $0 \%$ after about $15 \mathrm{~min}$. Adjusting the settings of the spin rotators could help to improve the polarization magnitude and lifetime. 
In addition to the polarization measurements discussed above, the allotted time allowed for two measurements to be taken for determining the spin tune. For these measurements, the target was first moved to the expected beam position. Then, the blue polarimeter DAQ was started manually, using the command line rather than using the standard application. After the DAQ was started, a single polarized proton bunch was injected into the ring. A horizontal target (Horizontal Target 1) was chosen for these measurements because it was significantly wider than the other targets. Figure 3.2 shows the measured number of events per bunch passing for this target. Using this target was necessary to provide the maximum possible statistics for the measurement.

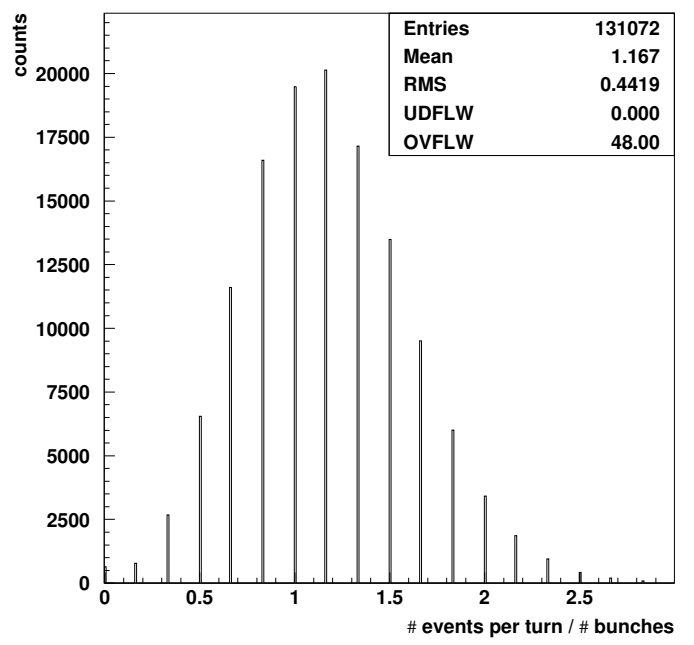

Figure 3.2: The number of detected events for each bunch passing the carbon target. The number of detected events is summed for each beam revolution, then divided by the number of bunches in the ring. This measurement was made using Horizontal Target 1 with 6 bunches in the blue ring. The beam intensity was approximately $1 \times 10^{11}$ protons per bunch. With a horizontal target, the target frame blocks events in detectors 2 and 5 , effectively decreasing the polarimeter acceptance by a third of the total acceptance. Despite the loss of acceptance, this target produces a much larger event rate than other targets due to its width.

To improve the statistical accuracy of the spin tune measurement, the asym- 
metries for each turn from the two individual measurements were summed. The asymmetries were then analyzed by applying the FFT to determine if they showed any signal of oscillating at a specific frequency. As shown in Figure 3.3, a clear signal is observed for the 1-4 asymmetry with a frequency of 0 . This so-called "DC component" at 0 frequency indicates that the beam is polarized, but it gives no information about the spin tune. Since the average beam polarization is seen in the 1-4 asymmetry, the spin tune signal is most likely expected to appear in the 3-6 asymmetry. The spin tune also is expected to have a value near 0.5. Figure 3.4 shows the 3-6 asymmetry for a range of frequencies near 0.5 . There is no clear spin tune signal seen.

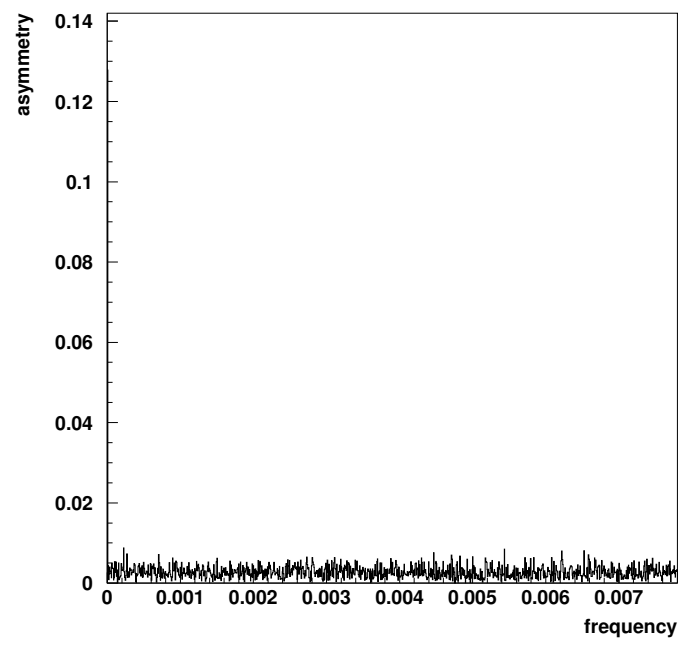

Figure 3.3: FFT amplitude of the asymmetry from polarimeter detectors 1 and 4. The asymmetry combines the data from two measurements. The FFT was applied to the first $2^{19}$ turns after the injection event. A significant signal is observed at frequency 0 . The amplitude is $\sim 0.128$. FFT analyses from later portions of the data show that this amplitude decreased over time, indicating depolarization of the beam.

Despite not producing a spin tune signal, this measurement technique shows promise. Detecting the injection signal with the polarimeter DAQ was successfully tested. Also, the DC component of the polarization vector was observed 


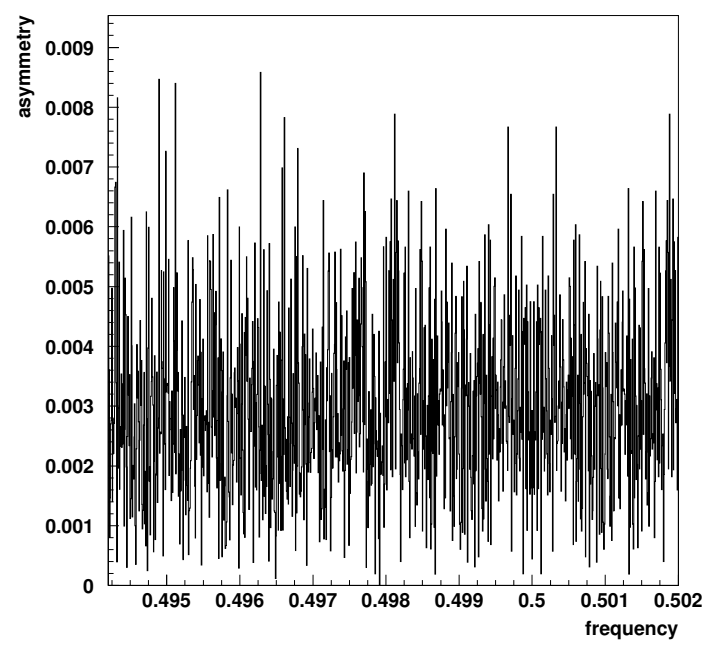

Figure 3.4: FFT amplitude of the asymmetry from polarimeter detectors 3 and 6. The FFT was applied to the first $2^{19}$ turns after the injection event. The spin tune is expected to produce an amplitude at a frequency near 0.5. However, no definite signal can be seen here.

using the FFT analysis. If the spin rotator settings can be optimized and the experiment can be repeated many times to increase statistics, then this measurement technique could prove to be an effective way to determine the spin tune at RHIC. 


\section{Conclusions}

The RHIC proton-carbon polarimeters played a vital role in the success of the 2006 run. The new advances, in particular, enhanced the day-to-day operations at RHIC. The online plots provided substantial information about each polarimeter measurement to the RHIC operators. This improved their knowledge of the polarimeter system and allowed them to quickly identify when there were problems with a measurement. The emittance scans with the polarimeters gave the RHIC operators and physicists an accessible way to frequently monitor the beam size. The tests of the spin tune measurement technique provided a foundation for future measurements that could help to optimize settings at RHIC. In addition to measuring the beam polarization, these improvements demonstrate the importance of the proton-carbon polarimeters at RHIC. 